\title{
Gender and Time Trend of Liver Cirrhosis Mortality and Its Estimated Avoidable Proportion in a Mountainous Province of Viet Nam from 2005 To 2018
}

\author{
Long Cong Nguyen ${ }^{1}$, Nghia Van Luu ${ }^{2}$, Nhung Thi Kim Ta ${ }^{3}$ and Ngoan Tran Le, \\ ${ }^{1}$ Gastroenterology \& Hepatology Center, Bach Mai Hospital, Hanoi, Viet Nam \\ ${ }^{2}$ Lang Son Provincial Center for Diseases Control (CDC), Lang Son city, Viet Nam \\ ${ }^{3}$ Department of Occupational Health, School of Preventive Medicine and Public Health, Hanoi Medical University, Viet Nam \\ ${ }^{4}$ Institute of Research and Development, Duy Tan University, Da Nang, Viet Nam \\ ${ }^{5}$ Department of Public Health, School of Medicine, International University of Health and Welfare, Japan
}

*Corresponding author: Ngoan Tran LE, Department of Public Health, School of Medicine, International University of Health and Welfare, Japan

ORCID:

Ngoan Tran Le: 0000-0001-7862-492X

Long Cong Nguyen: 0000-0002-5275-9014

\section{ARTICLE INFO}

Received: 慧 October 10, 2021

Published: November 05, 2021

Citation: Long Cong Nguyen, Nghia Van Luu, Nhung Thi Kim Ta, Ngoan Tran Le. Gender and Time Trend of Liver Cirrhosis Mortality and Its Estimated Avoidable Proportion in a Mountainous Province of Viet Nam from 2005 To 2018. Biomed J Sci \& Tech Res 39(5)-2021. BJSTR. MS.ID.006352.

Keywords: Liver Cirrhosis; Mortality Trend; Premature Death; Viet Nam

Abbreviations: CDC: Center for Diseases Control; CHS: Commune Health Stations ; ASR: Age-Standardized Rates

\begin{abstract}
Objectives: The aim was to estimate mortality rates, time trends by sex, and its estimated avoidable proportion of death due to cirrhosis in Lang son Province from 2005 to 2018.

Methods: We accessed a database of population-based mortality registration conducted yearly in 226 state health centers of 11 cities/districts of the Lang Son province to derive 2,612 death cases during 2005-2018. The variables of each case included the patient ID, age, sex, date, the cause of death, ICD-10 code. Mortality rates ratio and 95\% confidence interval (MRR, 95\%CI), age-standardized mortality rates (ASR), and an avoidable proportion of death were estimated.
\end{abstract}

Results: There was $88.5 \%$ of total premature deaths occurred under the age of 70 . The estimated ASR per 100,000 was remarkably higher in men (63.2) than women (7.3). When compared to women, the estimated avoidable proportion was $88.5 \%$ of 2,321 death cases in men. The estimated mortality rates ratios, the year 2018 versus 2005, were significantly increased in men, MRR (95\%CI): 1.367 (1.104, 1.693), $p=0.004$; but significantly decreased in women, MRR (95\%CI): 0.344 (0.177, 0.668), p=0.002.

Conclusion: The findings suggested that Liver Cirrhosis is a preventable disease and primary prevention and screening for the disease at an early stage would be highly needed to reduce premature deaths occurred in communities.

\section{Introduction}

During 1980-2017, Liver Cirrhosis was the major mortality of liver diseases worldwide [1]. The disease is chronic progressive liver fibrosis due to lifestyles and environmental factors [2]. In the initial stages, patients who suffered from liver cirrhosis are asymptomatic and the disease incidence rates are commonly underestimated [3]. Once clinical symptoms occur, the morbidity and premature death resulting from cirrhosis increase sharply [4]. Liver diseases due to chronic infection with hepatitis viruses [5-8] and high consumption of alcohol [9] have been observed to be common in Viet Nam from the last century to date. Harmful usage of alcohol, chronic infection with hepatitis viral infection, and smoking might be responsible for cirrhosis. Previous results have pointed out the major risk of 
cirrhosis was "Alcoholism" in many countries [10-13]. Based on these facts and figures, we have hypothesized that liver cirrhosis is a lifestyle-related health event and a possible avoidable disease. Patients suffering from liver cirrhosis need frequent medical care and their quality of life is affected [14]. Patients with liver cirrhosis have significantly increased morbidity and mortality [15]. However, few studies have been performed to address this public health problem in Viet Nam to date. This study aimed to estimate mortality rates, time trends by sex, and to estimate an avoidable proportion of death due to cirrhosis in men in Lang son Province from 2005 to 2018.

\section{Methods}

The study was performed in Lang Son province, a mountain highland province in northern Viet Nam. The study population is bordering Guangxi province in China and other provinces in Viet Nam. It was a population-based mortality registration operated by the Lang Son Center for Diseases Control (CDC) that covered all 226 communes of 11 cities/districts of the province, with a population of 790,500 people in 2018 [16]. In Viet Nam, the national mortality registration systems have been started from 1992 that was following the guideline by the Ministry of Health to report the causes of death from all health facilities nationwide. From 1992 to date, Lang Son is one of all 63 cities/provinces of the country to actively conduct mortality registration based on the medical records available at 226 state commune health stations (CHS), 10 district general hospitals, 9 district out-patient clinics, and 3 provincial general and specialized hospitals. The head of the CHS is a person in charge of the monthly report of all death cases that occurred at his/her communes in a mortality registration book, named A6-mortality book that was designed by the Ministry of Health. The variables included name, age, sex, ethnicity, address, occupation, date, and cause of death, place of death occurred (at a health facility, home, other), and attended emergency care at the endpoint (Yes/no), and the name of the reporter.

From 2005 to date, the Lang Son CDC has collected data from all 226 CHS using the data collection form in the hard copy of "Mortality Registration" for six variables of name, age, sex, occupation, date, and cause of death for every year. The additional guidelines to determine the causes of deaths, including the immediate cause of death, a contributing cause of death, and the underlying cause of death, and its ICD-10 code, were sent with the designed form of "Mortality Registration". The average population number by sex has also been collected by this form for every year from 2005 to 2018 . A yearly population structure by age groups of $(0-9,10-19,20-29,30$ $39,40-49,50-59,60-69,70-79,80+$ ) by sex was estimated using the results of the National Census conducted in 1999, 2009, and 2019.
The collected variables of each death case were inputted in Excel for each district for each year. The ID and ICD-10 code for the cause of death was made for each case. In this way, a database of mortality from 2005 to 2018, missing data for 2009-2010, was made by the Lang Son CDC. The dataset included 49,253 reported death cases due to all causes $[16,17]$.

We accessed to Lang Son database of mortality during 20052018, missing data for 2009-2010, to derive variables of case's ID, age, sex, date, and cause of death, ICD-10 code. We found ICD-10 code K74 for Liver Cirrhosis and a total number of 2,612 cases for the present study. We also derived the average number of population by years and by communes for further estimate number of person-year for the final analysis. We calculated the mortality rates ratio with a 95\% confidence interval (MRR, 95\%CI) using regression analysis, adjusted for age groups of 10-year intervals $(0-9,10-19,20-29,30-39,40-49,50-59,60-69,70-79,80+)$ and sex. Age-standardized rates (ASR) per 100,000 person-years were calculated using the world population structure as the standard. We calculated the avoidable proportion of deaths in men by the formulation of ((a-b)*100/a (a: ASR in men; b: ASR in women). The Board of Ethics in Bio-Medical Research at University of Medicine and Pharmacy at Ho Chi Minh City \#106/UMP-BOARD, on 20 March 2019 and Hanoi Medical University Review Board in Bio-Medical Research \# 61/HMURB, on 25 November 2008 have approved the present research protocol.

\section{Results}

Overall, a total of 2,612 deaths were registered among residents in Lang Son Province from 2005 to 2018. Table 1 shows the results of deaths were 2,321 in men and 291 in women. Men had a higher age-adjusted mortality rate (ASR-WHO 63.2 per 100,000) due to cirrhosis compared to women (ASR-WHO 7.3 per 100,000), giving man to women ratio: 8.7/1 (63.2/7.3). The estimated men to women ratio were sharply increased from $4.9 / 1$ (49.1/10.1) in 2005 to $18.6 / 1(63.1 / 3.5)$ in 2018. Compared to women, the estimated avoidable proportion of death from Liver Cirrhosis in men was $((63.2-7.3) * 100) / 63.2=88.5 \%$. There was $88.5 \%$ of total premature deaths occurred under the age of 70 , Table 1 . The agestandardized death rate increased gradually according to WHOASR from 27.7 per 100,000 to 33.4 per 100,000 person-years for both genders from 2005 to 2018, respectively. The estimated proportion of deaths due to Liver Cirrhosis was 5.3\% (2,612 cases of Liver Cirrhosis vs. 49,253 total death cases) in both genders. Overall death due to the disease, men, and women combined, was significantly increased from 2005 to 2018. After adjusting for age and sex, per-year increment was significantly seen, MRR (95\%CI): 1.019 (1.009, 1.028), $\mathrm{p}=0.001$ (Table 2). 
Table 1: Mortality due to Liver Cirrhosis by sex during 2005-2018 in Lang Son province.

\begin{tabular}{|c|c|c|c|c|c|c|c|}
\hline Sex & Year & Cause & Total & Crude rate \& & ASR @ & \%<70 \# & WHO \$ \\
\hline Men & $2005-18$ & K74 & 2,321 & 51.4 & 57.8 & 91.3 & 63.2 \\
\hline Women & $2005-18$ & K74 & 291 & 6.4 & 6.6 & 66.3 & 7.3 \\
\hline Men and women & $2005-18$ & K74 & 2,612 & 28.8 & 30.3 & 88.5 & 33.1 \\
\hline
\end{tabular}

Note: Missing data for the year 2009-2010; \&: Crude rate per 100,000 person-years; \$: Age-standardized rate per 100,000 person-years using the World Health Organization standard population for 2000-2025; \# Proportion of death cases aged under 70 year-olds. @: Age-standardized rate per 100,000 person-years using the SEGI World standard population (in the 1960s). Men to women ratio (ASR$\mathrm{WHO})=8.7(63.2 / 7.3)$. The estimated proportion of death from Liver Cirrhosis in men was $\left\{(63.2-7.3)^{*} 100\right\} / 63.2=88.5 \%$

Table 2: Mortality due to Liver Cirrhosis in both genders by year from 2005 to 2018 in Lang Son province.

\begin{tabular}{|l|c|c|c|c|c|c|}
\hline Year & Case & Crude rate \& & \%<70 \# & WHO-ASR \$ & MRR (95\%CI) \$\$ & p \\
\hline 2005 & 173 & 24.1 & 85.5 & 27.7 & 1.00 (reference) & 0.484 \\
\hline 2006 & 163 & 22.3 & 85.3 & 26.0 & $0.926(0.748,1.147)$ & 0.493 \\
\hline 2007 & 190 & 25.9 & 84.2 & 29.5 & $1.075(0.875,1.320)$ & 0.653 \\
\hline 2008 & 188 & 25.2 & 92.6 & 29.4 & $1.049(0.853,1.289)$ & 0.004 \\
\hline 2011 & 235 & 32.1 & 83.4 & 37.0 & $1.333(1.096,1.623)$ & 0.026 \\
\hline 2012 & 225 & 30.1 & 86.2 & 34.2 & $1.253(1.028,1.528)$ & 0.002 \\
\hline 2013 & 243 & 33.0 & 89.7 & 37.9 & $1.370(1.127,1.665)$ & $<0.001$ \\
\hline 2014 & 269 & 34.5 & 90.3 & 39.8 & $1.432(1.183,1.734)$ & 0.008 \\
\hline 2015 & 246 & 31.3 & 85.8 & 35.5 & $1.230(1.070,1.579)$ & 0.005 \\
\hline 2016 & 249 & 31.7 & 89.6 & 37.1 & $1.319(1.087,1.602)$ & 0.387 \\
\hline 2017 & 209 & 26.3 & 93.3 & 30.3 & $1.093(0.894,1.337)$ & 0.107 \\
\hline 2018 & 222 & 28.3 & 95.0 & 32.4 & $1.178(0.965,1.437)$ & 2 \\
\hline
\end{tabular}

Note: Missing data for the year 2009-2010; the estimated proportion of deaths due to Liver Cirrhosis was 5.30\% $(2,612$ cases of Liver Cirrhosis vs. 49,253 total cases), both genders. \$\$ adjusted for age group (0-9, 10-19, 20-29, 30-39, 40-49, 50-59, 60-69, 70-79, 80+) and sex. Per-year increment MRR (95\% CI): 1.019 (1.009, 1.028), p<0.001. \& Crude rate per 100,000 person-years; \$ Age-standardized rate per 100,000 person-years using the World Health Organization standard population for 2000-2025; \# Proportion of death cases aged under 70 year-olds. When combined all cases from 2005-2018, both genders, WHO-ASR: 31.3 per 100,000 person-years.

In men, the estimated proportion of deaths due to Liver Cirrhosis was $7.4 \%$ (2,321 cases of Liver Cirrhosis vs. 31,262 total cases). The age-standardized mortality rates per 100,000 were increased from 49.1 in 2005 to 65.1 in 2018. The estimated mortality rates ratios, the year 2018 versus 2005, were significantly increased, MRR (95\%CI): 1.367 (1.104, 1.693), p=0.004. During this period, per-year increment was significantly observed, MRR (95\%CI): 1.028 (1.018, 1.038), $p=0.001$ (Table 3). In Women, the estimated proportion of deaths due to Liver Cirrhosis was lower (1.6\%, 291 cases of Liver Cirrhosis vs. 17,990 total death cases) than in men $(7.4 \%, 2,321$ cases of Liver Cirrhosis vs. 31,262 total death cases). For a time trend, in contrast, death due to the disease was significantly decreased in women from 2005 to 2018. The estimated mortality rates ratios, the year 2018 versus 2005, were significantly decreased, MRR (95\%CI): 0.344 (0.177, 0.668), $\mathrm{p}=0.002$. During this period, per-year decrement was significantly seen, MRR (95\%CI): 0.948 (0.923, 0.974), p=0.001 (Table 4). By age-specific, the estimated mortality rates per 100,000 in men were much higher than in women for all age groups, especially for the age group (50-59), 193.5 versus 13.2, respectively (Figure 1).

Table 3: Mortality due to Liver Cirrhosis in men by year from 2005 to 2018 in Lang Son province.

\begin{tabular}{|l|c|c|c|c|c|c|}
\hline Year & Case & Crude rate \& & $\mathbf{\%}<\mathbf{7 0 ~ \#}$ & WHO-ASR \$ & MRR (95\%CI) \#\# & p \\
\hline 2005 & 141 & 39.4 & 86.5 & 49.1 & 1.00 (reference) & 0.748 \\
\hline 2006 & 138 & 37.9 & 87.0 & 47.6 & $0.962(0.761,1.217)$ & 0.216 \\
\hline 2007 & 166 & 45.4 & 88.6 & 55.3 & $1.152(0.920,1.442)$ & 0.574 \\
\hline 2008 & 156 & 42.7 & 95.5 & 51.3 & $1.068(0.850,1.341)$ & 0.002 \\
\hline 2011 & 201 & 55.1 & 88.6 & 69.0 & $1.400(1.128,1.736)$ & 0.004 \\
\hline 2012 & 200 & 53.9 & 89.0 & 65.4 & $1.367(1.102,1.697)$ & \\
\hline
\end{tabular}




\begin{tabular}{|c|c|c|c|c|c|c|}
\hline 2013 & 221 & 60.2 & 92.3 & 73.5 & $1.530(1.238,1.890)$ & $<0.001$ \\
\hline 2014 & 241 & 62.0 & 92.9 & 75.9 & $1.575(1.279,1.940)$ & $<0.001$ \\
\hline 2015 & 216 & 55.2 & 88.4 & 67.3 & $1.401(1.133,1.732)$ & 0.002 \\
\hline 2016 & 234 & 59.9 & 91.5 & 75.8 & $1.522(1.235,1.876)$ & $<0.001$ \\
\hline 2017 & 197 & 49.8 & 94.9 & 61.6 & $1.264(1.019,1.570)$ & 0.033 \\
\hline 2018 & 210 & 53.9 & 97.6 & 65.1 & $1.367(1.104,1.693)$ & 0.004 \\
\hline
\end{tabular}

Note: Missing data for the year 2009-2010; the estimated proportion of deaths due to Liver Cirrhosis was 7.42\% (2,321cases of Liver Cirrhosis vs. 31,262 total cases) in men. \#\# adjusted for age group (0-9, 10-19, 20-29, 30-39, 40-49, 50-59, 60-69, 70-79, 80+). Per-year increment MRR (95\%CI): 1.028 (1.018, 1.038), p<0.001. \& Crude rate per 100,000 person-years; \$ Age-standardized rate per 100,000 person-years using the World Health Organization standard population for 2000-2025; \# Proportion of death cases aged under 70 year-olds. When combined all cases from 2005-2018 in men, WHO-ASR: 63.2 per 100,000 person-years.

Table 4: Mortality due to Liver Cirrhosis in women by year from 2005 to 2018 in Lang Son province.

\begin{tabular}{|c|c|c|c|c|c|c|}
\hline Year & Case & Crude rate \& & $\%<70 \#$ & WHO-ASR \$ & MRR (95\%CI) \#\# & $\mathbf{p}$ \\
\hline 2005 & 32 & 8.9 & 81.3 & 10.1 & 1.00 (reference) & \\
\hline 2006 & 25 & 6.8 & 76.0 & 7.9 & $0.768(0.455,1.296)$ & 0.323 \\
\hline 2007 & 24 & 6.5 & 54.2 & 7.3 & $0.734(0.432,1.246)$ & 0.252 \\
\hline 2008 & 32 & 8.5 & 78.1 & 10.2 & $0.965(0.591,1.575)$ & 0.886 \\
\hline 2011 & 34 & 9.2 & 52.9 & 9.8 & $1.043(0.643,1.690)$ & 0.865 \\
\hline 2012 & 25 & 6.7 & 64.0 & 7.8 & $0.753(0.446,1.270)$ & 0.287 \\
\hline 2013 & 22 & 5.9 & 63.6 & 7.0 & $0.671(0.390,1.154)$ & 0.149 \\
\hline 2014 & 28 & 7.1 & 67.9 & 8.4 & $0.806(0.485,1.338)$ & 0.404 \\
\hline 2015 & 30 & 7.6 & 66.7 & 8.3 & $0.857(0.521,1.410)$ & 0.544 \\
\hline 2016 & 15 & 3.8 & 60.0 & 4.2 & $0.430(0.233,0.793)$ & 0.007 \\
\hline 2017 & 12 & 3.0 & 66.7 & 3.5 & $0.339(0.175,0.659)$ & 0.001 \\
\hline 2018 & 12 & 3.0 & 50.0 & 3.5 & $0.344(0.177,0.668)$ & 0.002 \\
\hline
\end{tabular}

Note: Missing data for the year 2009-2010; the estimated proportion of deaths due to Liver Cirrhosis was $1.62 \%$ (291 cases of Liver Cirrhosis vs. 17,990 total cases) in women. \#\# adjusted for age group $(0-9,10-19,20-29,30-39,40-49,50-59,60-69,70-79,80+)$. Per-year increment MRR (95\%CI): 0.948 (0.923, 0.974), p<0.001. \& Crude rate per 100,000 person-years; \$ Age-standardized rate per 100,000 person-years using the World Health Organization standard population for 2000-2025; \# Proportion of death cases aged under 70 year-olds. When combined all cases from 2005-2018 in women, WHO-ASR: 7.3 per 100,000 person-years.

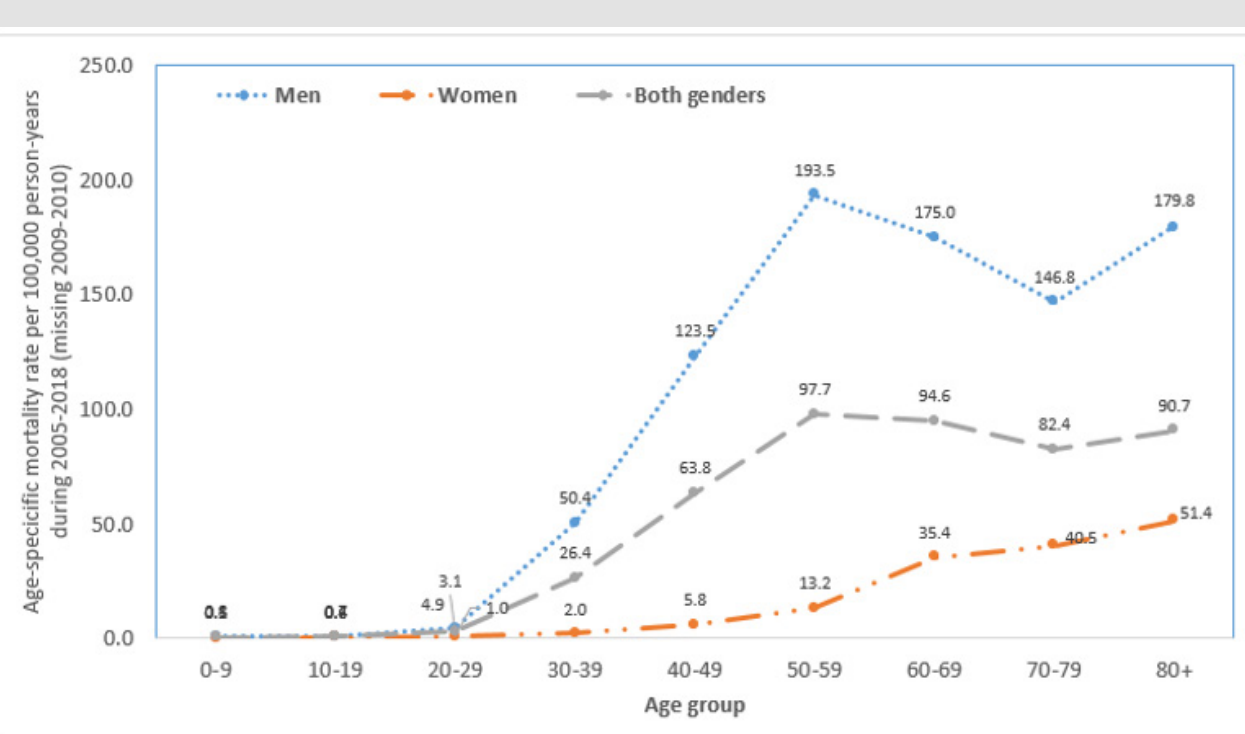

Figure 1: Age-specific mortality rate per 100,000 person-years by sex during 2005-2018 due to Liver Cirrhosis. Note: Missing data for the year 2009-2010; 


\section{Discussion}

We observed a divergence over time from 2005 to 2018 between men (statistically significant increase) and women (statistically significant decrease) in a community in the mountainous province of Lang Son in northern Viet Nam. When compared to women, the estimated avoidable proportion of death due to the disease was about $88.5 \%$ in men. There was serious premature death, as high as $88.5 \%$ for both genders ( $91.3 \%$ in men and $66.3 \%$ in women). The findings suggested that Liver Cirrhosis is a preventable disease and performing primary prevention in the community to reduce premature death would be highly needed. Harmful usage of alcohol by men living in the Lang Son province might be the major risk factor of developing Liver Cirrhosis. An estimated annual amount of pure alcohol consumption in 2010 was 12.1 liters and 0.2 liters in men and women, respectively [18]. This big gap between men and women can be consistent with the significantly higher risk of death from Liver Cirrhosis in men than in women. Between men and women, there were no significant differences for both Hepatitis B virus, HBsAg positive (Odds ratio and $95 \%$ confidence interval, men versus women: $0.76(0.54-1.01))$ and Hepatitis $C$ virus infection (Odds ratio and 95\% confidence interval, men versus women: 1.73 $0.41-7.28)[5,19]$.

Therefore, viral Hepatitis infection could not be explained for the big gap between genders in the risk of death from this disease. The present findings support our hypothesis that liver cirrhosis is a lifestyle-related health event and a possible avoidable disease. The results warrant further observational studies to conclude the association between alcoholism and Liver Cirrhosis in the disadvantaged areas in Viet Nam with emphasis on primary prevention of the disease. There was rarely alcohol drinking $(0.2 \%)$ and tobacco smoking in Vietnamese women aged $15+(1.7 \%)$ in 2010 [18] and it was a similar observation in the 1990s [20]. The estimated prevalence of Hepatitis $C$ virus infection was very low $(0.5 \%)$ in rural areas [19]. There was also a very low prevalence $(4.8 \%)$ of overweight among Vietnamese women. Therefore, Vietnamese women will be minimized risk of Liver Cirrhosis caused by alcoholism, smoking, Hepatitis C virus infection, and obesity. From 2005 to 2018, nutrition and diet, living and working environments have been improving which might be explained why there was a significantly decreased trend of mortality from the disease in the study population. In contrast, a significant increasing trend in men might be related to the increasing prevalence of harmful usage of alcohol from $25.5 \%$ in 2004 to $35.0 \%$ in 2013 [21].

The present study has several strengths, including long time of mortality registration from 2005 to 2018 to examine time trend of the disease; a large number of mortality cases was reported for both men and women; the covered study population was for the entire
Lang Son province with good healthcare systems from communes to district and provincial health facilities; mortality registration was actively and yearly done by professional health workers; the average population by each of 226 communes was updated for every year that allows us to estimate the age-standardized mortality rates by genders and for each year [16,17]. In addition, the quality of mortality registration in Viet Nam has been validated [22-24] to be performed well which will be feasible, reliable, and practical to develop annual local and national mortality databases of mortality to identify priority public health problems and to create planning timely actions in general and in controlling Liver Cirrhosis in particular. Based on these favorable research conditions, we can observe a real problem of the disease and add some new pieces of knowledge for further elimination of this preventable health threat in our society.

Liver Cirrhosis was a serious public health problem at the disadvantaged highland in Viet Nam of the present study population and that was consistent with findings in the world. There were 1.32 million deaths due to Liver Cirrhosis reported in 2017, with 440,000 deaths in women and 883,000 deaths in men. The burden of disease has been increased from 1990 to 2017. That is, the estimated deaths due to Liver Cirrhosis were responsible for about $2.4 \%$ of total deaths in the world in 2017 compared with $1.9 \%$ in 1990 [25]. In this study, we found that mortality due to cirrhosis was $5.3 \%$ of total deaths during 2005-2018 in the Lang Son province that was over doubled than the world estimation. There has been no systematic nationwide screening for Liver Cirrhosis in Viet Nam, especially in the mountain areas, and no nationwide comprehensive response to the disease.

The present study has certain limitations that include i) missing data for the year 2009-2010; ii) there is no available data of histopathological confirmation of Liver Cirrhosis, and iii) there is not the available data of lifestyle and environmental factors related to the disease. Despite these limitations, the present findings warrant further studies to identify risk factors for implementing primary prevention and secondary prevention in the community.

\section{Source(s) of Support}

There was no support.

\section{Conflicting Interest}

There are no conflicts of interest to disclose.

\section{Acknowledgment}

We are grateful to all staff of the Lang Son's Health facilities involved in data collection, supervision, monitoring, and data management. There were no other conflicts of interest. All authors had input into the final version of the paper. 


\section{References}

1. (2018) GBD 2017 Causes of Death Collaborators. Global, regional, and national age-sex-specific mortality for 282 causes of death in 195 countries and territories, 1980-2017: a systematic analysis for the Global Burden of Disease Study 2017. Lancet 392(10159): 1736-1788.

2. Anthony PP, Ishak KG, Nayak NC, Poulsen HE, Scheuer PJ, et al. (1978) The morphology of cirrhosis. Recommendations on definition, nomenclature, and classification by a working group sponsored by the World Health Organization. J Clin Pathol 31(5): 395-414.

3. Fleming KM, Aithal GP, Card TR, West J (2010) The rate of decompensation and clinical progression of disease in people with cirrhosis: a cohort study. Aliment Pharmacol Ther 32(11-12): 1343-50.

4. D’Amico G, Garcia-Tsao G, Pagliaro L (2006) Natural history and prognostic indicators of survival in cirrhosis: a systematic review of 118 studies. J Hepatol 44(1): 217-231.

5. Nguyen VT, McLaws ML, Dore GJ (2007) Highly endemic hepatitis B infection in rural Vietnam. J Gastroenterol Hepatol 22(12): 2093-100.

6. Tran HT, Ushijima H, Quang VX, Phuong N, Li TC, et al. (2003) Prevalence of hepatitis virus types B through $\mathrm{E}$ and genotypic distribution of HBV and HCV in Ho Chi Minh City, Vietnam. Hepatol Res 26(4): 275-280.

7. Hipgrave DB, Nguyen TV, Vu MH, Hoang TL, Do TD, et al. (2003) Hepatitis $B$ infection in rural Vietnam and the implications for a national program of infant immunization. Am J Trop Med Hyg 69(3): 288-294.

8. Nakata S, Song P, Duc DD, Nguyen HQ, Murata K, et al. (1994) Hepatitis C and $\mathrm{B}$ virus infections in populations at low or high risk in Ho Chi Minh and Hanoi, Vietnam. J Gastroenterol Hepatol 9(4): 416-419.

9. Huu Bich T, Thi Quynh Nga P, Ngoc Quang L, Minh HV, NG N, et al. (2009) Patterns of alcohol consumption in diverse rural populations in the Asian region. Glob Health Action, p. 2.

10. Wiegand J, Berg T (2013) The etiology, diagnosis, and prevention of liver cirrhosis: part 1 of a series on liver cirrhosis. Dtsch Arztebl Int 110(6): 85-91.

11. Goncalves PL, Zago-Gomes Mda P, Marques CC, Mendonca AT, Goncalves CS, et al. (2013)Etiology of liver cirrhosis in Brazil: chronic alcoholism and hepatitis viruses in liver cirrhosis diagnosed in the state of Espirito Santo. Clinics (Sao Paulo) 68(3): 291-295.

12. Bell BP, Manos MM, Zaman A, Norah Terrault, Ann Thomas, et al. (2008) The epidemiology of the newly diagnosed chronic liver disease in gastroenterology practices in the United States: results from populationbased surveillance. Am J Gastroenterol 103(11): 2727-36; quiz 37.

ISSN: 2574-1241

DOI: 10.26717/BJSTR.2021.39.006352

Ngoan Tran Le. Biomed J Sci \& Tech Res

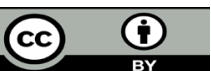

his work is licensed under Creative Commons Attribution 4.0 License

Submission Link: https://biomedres.us/submit-manuscript.php
13. Mendez-Sanchez N, Aguilar-Ramirez JR, Reyes A, Dehesa M, Juorez A, et al. (2004) Etiology of liver cirrhosis in Mexico. Ann Hepatol 3(1): 30-33.

14. Ge PS, Runyon BA (2016) Treatment of Patients with Cirrhosis. N Engl J Med 375(8): 767-77.

15. Scaglione S, Kliethermes S, Cao G, Shoham D, Durazo R, et al. (2015) The Epidemiology of Cirrhosis in the United States: A Population-based Study. J Clin Gastroenterol 49(8): 690-696

16. Nghia LV (2020) Status of injury mortality, population-based injury mortality registration in Lang Son province, Vietnam during 2016-2018. Center for Disease Control of Lang Son province: Lang Son Provincial Health Department.

17. Nghia LV (2017) Study on work-related mortality in Lang Son province during 2011-2014 [Master Thesis]. Department of Occupational Health. Hanoi Medical University.

18. (2014) WHO (2014) World Health Organization - Cancer Country Profiles, 2014: Viet Nam.

19. Nguyen VT, McLaws ML, Dore GJ (2007) Prevalence and risk factors for hepatitis C infection in rural northern Vietnam. Hepatol Int 1(3): 387393.

20. Jenkins CN, Dai PX, Ngoc DH, Kinh HV, Hoang TT, et al. (1997)Tobacco use in Vietnam. Prevalence, predictors, and the role of the transnational tobacco corporations. JAMA 277(21): 1726-1731.

21. Lincoln M (2016) Alcohol and drinking cultures in Vietnam: A review. Drug Alcohol Depend 159: 1-8.

22. Hong TT, Hoa NP, Walker SM, Hill PS, Rao C (2018) Completeness and reliability of mortality data in Viet Nam: Implications for the national routine health management information system. PLoS One 13(1): e0190755.

23. Thuong NV, Minh LQ, Stevenson M, Linh LT, Ngoan LT (2017) Evaluation of a Population-based Intervention to Improve the Vietnamese Mortality Reporting System. Southeast-Asian J of Sciences 5(2): 194-204.

24. Stevenson M, Hung DV, Hoang TH, Mai Anh L, Nguyen Thi Hong T, et al. (2015) Evaluation of the Vietnamese A6 Mortality Reporting System: All-Cause Mortality. Asia Pac J Public Health 27(7): 733-742.

25. (2020) GBD 2017 Cirrhosis Collaborators. The global, regional, and national burden of cirrhosis by cause in 195 countries and territories, 1990-2017: a systematic analysis for the Global Burden of Disease Study 2017. Lancet Gastroenterol Hepatol 5(3): 245-266.

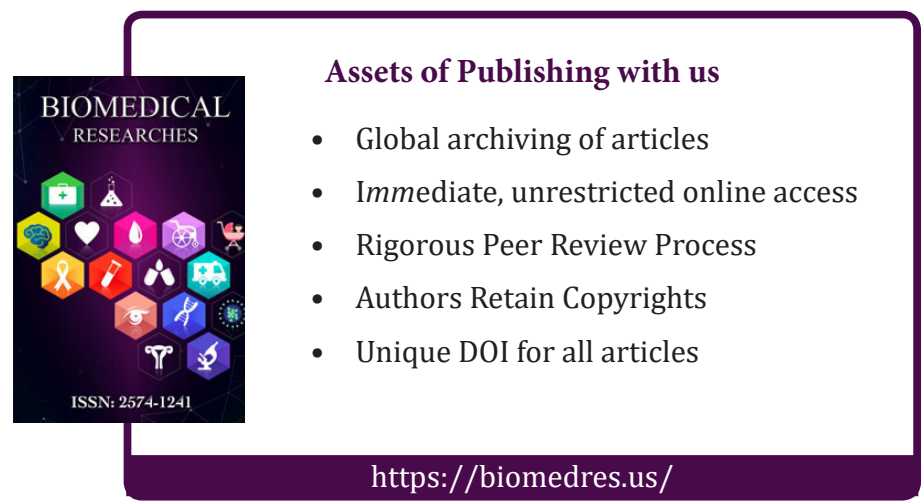

